\title{
Modeling and Performance Analysis of Manufacturing Execution System Based on Petri Net
}

\author{
Mo Taiping ${ }^{1, *}$ and Zhao Peisi ${ }^{2}$ \\ ${ }^{1}$ School of Mechano-electronic Engineering, Xidian University, Xi'an 710071, China; ${ }^{2}$ School of Electronic Engineering \\ \& Automation, Guilin University of Electronic Technology, Guilin 541004, China
}

\begin{abstract}
The current Manufacturing Execution System (MES) has many weaknesses, such as poor generality, weak reconfigurability and difficult performance analysis. In order to solve these problems, a functional decomposition diagram was designed based on the functional requirements of MES, and a general petri net model was also established. The complexity and junction features of the model were analyzed based on Petri nets, and the network core layer and system reliability were gotten after the model was transformed to the undirected network diagram. Finally, the best system scheme for different selections was given based on the analysis of reliability.
\end{abstract}

Keywords: Complexity, manufacturing execution system, modeling, Petri nets, reliability.

\section{INTRODUCTION}

With the application fields expansion of information system, the scope and complexity of the system is increased greatly. The system includes a large amount of problems upon engineering, technology and efficient allocation of resources. It is different to deal with the top design and make sufficient research, analysis and discussion by virtue of experience. The MES manages the whole manufacturing system according to the production plan, material management strategy, quality standard, quality inspection method and the cost accounting method established by the management department. In addition, it supervises and controls the various links in real-time, so as to achieve the integrative, informationalized and transparent effect on manufacturing process management. However, the current MES has poor generality in the industry, but also there is no convenient way to analyze the complexity and feasibility during the early stage of MES development. In order to solve these problems, a general MES model should be established.

As a formalized description tool, Petri net [1-8] provides formal modeling methods base on graphics and mathematics, and it plays a key role in the process of modeling and performance analysis of complex network system, so the Petri model is estimated in this paper to solve the MES problems.

\section{KEY FUNCTION OF MES}

MES is a process oriented software management systems, and it plays key role in the three-layer enterprise integration model and it connects the Enterprise Resource Planning Systems (ERP) in the upper layer and the
Process Control Systems (PCS) in the lower layer. Manufacturing Execution System Association (MESA) defines functions of the MES function models as: a detail process scheduling, resource allocation and state management, production element allocation, process management, human resource management, maintenance management, quality management, document control, product tracking and product inventory management, performance analysis and data acquisition functions [5]. The MES core functions include production scheduling management, product tracking, material management, process quality management, product storage and shipping and basic production data collection and analysis.

\section{THE PETRI NET MODEL OF MES}

According to the above-mentioned functions and structure of MES, MES's overall business processes are as follows:

1) Make the corresponding plans according to the sales plan, production process, manufacturing resources and the production mode;

2) Manage the workshop materials according to the storage management strategy, material management mode of production, productive task, input and output information;

3) Develop appropriate online quality management strategy based on quality standards and test methods;

4) Manage production costs based on hours of work, production methods, basic statistics and cost accounting methods;

5) Summarize the system data, including the production statements, material statements, quality statements and production cost statements;

6) Submit statistics and aggregated data, analyze production status. 


\subsection{System Interface Specification}

MES external environment includes ERP, Supply Chain Management (SCM), Product Data Management (PDM) and PCS. According to the business process above, the interface elements should include:

$\mathrm{I}_{1}$ : Marking plan; $\mathrm{I}_{2}$ : Data for each unit; $\mathrm{I}_{4}$ : Production process; $\mathrm{I}_{5}$ : Manufacturing resource; $\mathrm{I}_{6}$ : Mode of production; $\mathrm{I}_{7}$ : Finished goods inventory report; $\mathrm{I}_{8}$ : Quality standards; $\mathrm{I}_{9}$ : Detection method; $\mathrm{I}_{10}$ : Cost accounting methods; $\mathrm{I}_{11}$ : Production report; $\mathrm{I}_{12}$ : Material inventory report; $\mathrm{I}_{13}$ : Quality report; $\mathrm{I}_{14}$ : Production cost report; $\mathrm{I}_{15}$ : Plan reports; $\mathrm{I}_{16}$ : Plan change notice; $\mathrm{I}_{17}$ : Device report

MES interface is described in Fig. (1).

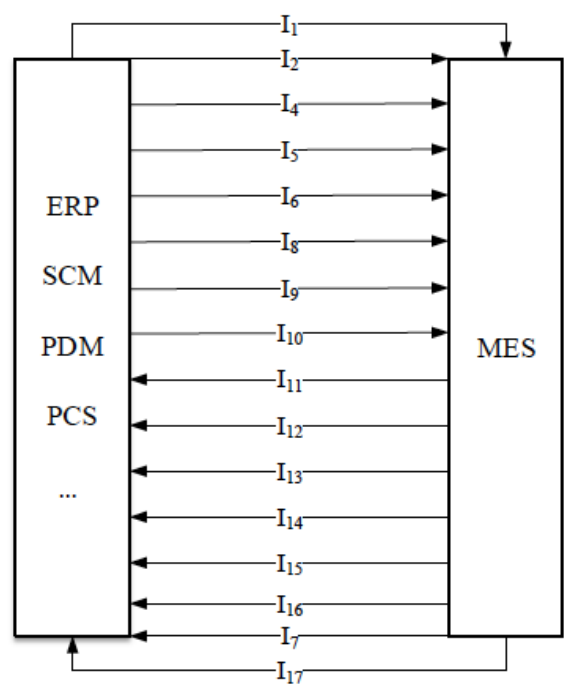

Fig. (1). MES interface description.

\subsection{Definition of MES Function Module}

Function is one of the basic elements of an information system model. During the design process of information sys- tem, the system implementation objectives must be clear. The objectives can be described with the function definition. According to MES business flow diagram, the MES function model can be defined as shown in Fig. (2).

Assumes that the leaf node of the system basic function represents a set $T_{S}$, According to the MES function models from Fig. (2), $T_{S}$, can be described as follows:

$$
\begin{aligned}
& T_{S}=\left\{t_{S 11}, t_{S 12}, t_{S 13}, t_{S 21}, t_{S 22}, t_{S 23}, t_{S 24}, t_{S 31}, t_{S 32}, t_{S 33},\right. \\
& t_{S 41}, t_{S 42}, t_{S 43}, t_{S 441}, t_{S 442}, t_{S 443}, t_{S 444}, t_{S 45}, t_{S 51}, t_{S 52}, \\
& \left.t_{S 53}, t_{S 54}, t_{S 6}, t_{S 71}, t_{S 72}, t_{S 73}, t_{S 74}, t_{S 81}, t_{S 82}\right\}
\end{aligned}
$$

\subsection{Internal Resource Constraints of MES}

Information resources is mainly the data, information carrier and related equipment for system operation. In order to improve the utilization of information resources and to facilitate the management and maintenance of information resources, it is necessary to configure all kinds of information resources in the system reasonably.

\subsubsection{The Definition of Information Resource set $P_{S}$}

Based on MES process, the main information resources are as follows:

$P_{11}$ : Operational plan; $P_{21}$ : Production data statistics; $P_{22}$ : Product login information;

$P_{23}$ : Product offline information; $P_{25}$ : Production data of workshop;

$P_{31}$ : Flaw classification information; $P_{32}$ : Flaw classification list; $P_{33}$ : Quality statistics; card;

$P_{41}$ : Equipment information; $P_{43}$ : Equipment information

$P_{44}$ : Equipment check application form; $P_{45}$ : Equipment check statements;

$P_{46}$ : Equipment operating state information; $P_{48}$ : Equipment status report;

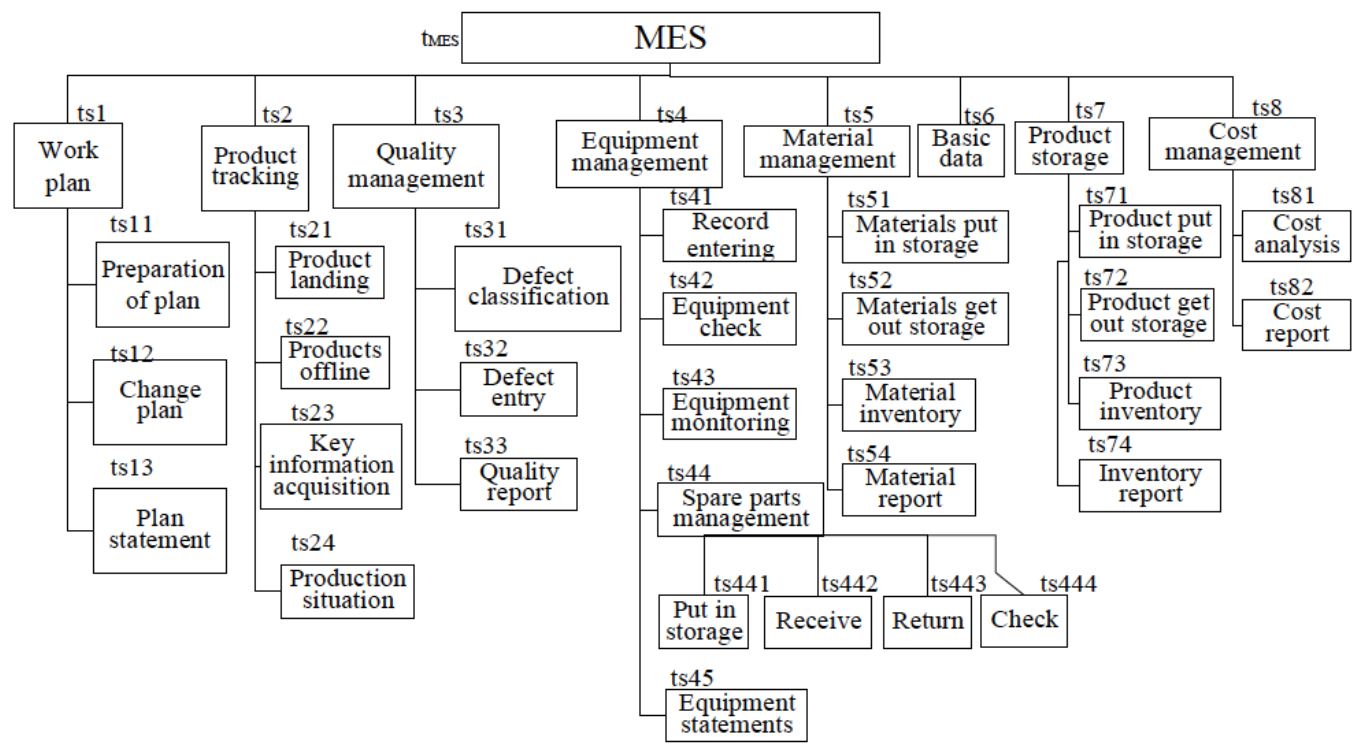

Fig. (2). Functional decomposition diagram of MES. 
$P_{51}$ : Material management strategy; $P_{52}$ : Material inventory information;

$P_{53}$ : The output material documents; $P_{54}$ : The warehouse warrant and delivery order of material;

$P_{57}$ : Goods Receiving Note; $P_{61}$ : Spare parts acceptance application form;

$P_{62}$ : Information of spare parts being used; $P_{63}:$ Spare parts inventory;

$P_{64}$ : The warrant and delivery order of spare parts store;

$P_{71}$ : Warehouse warrant of finished products; $P_{72}$ : Inventory information of finished goods;

$P_{73}$ : Delivery order of finished goods; $P_{74}$ : Product inventory list;

$P_{81}$ : Cost analysis and statistics.

Thereby we obtain the set of information resources $P_{S}$ :

$P_{S}=\left\{P_{11}, P_{21}, P_{22}, P_{23}, P_{25}, P_{31}, P_{32}, P_{33}, P_{41}, P_{43}, P_{44}, P_{45}, P_{46}\right.$,

$\left.P_{48}, P_{51}, P_{52}, P_{53}, P_{54}, P_{57}, P_{61}, P_{62}, P_{63}, P_{64}, P_{71}, P_{72}, P_{73}, P_{74}, P_{81}\right\}+$

$\left\{I_{1}, I_{2}, I_{4}, I_{5}, I_{6}, I_{7}, I_{8}, I_{9}, I_{10}, I_{11}, I_{12}, I_{13}, I_{14}, I_{15}, I_{16}, I_{17}\right\}$

\subsubsection{The Definition of Resource Constraints set $P_{T}$ for $T_{S}$}

The set of the constrained subset of the used resource by each function model is called the system information resource constraint set [8], which is expressed by $T_{S}$. Based on the system model, we get the system information resource constraint set $P_{T}$ :

$$
\begin{aligned}
& P_{T}=\left\{P_{t s 11}, P_{t s 12}, P_{t s 13}, P_{t s 21}, P_{t s 22}, P_{t s 23}, P_{t s 24}, P_{t s 31}, P_{t s 32}, P_{t s 33}, P_{t s 41},\right. \\
& P_{t s 42}, P_{t s 43}, P_{t s 441}, P_{t s 442}, P_{t s 443}, P_{t s 444}, P_{t s 45}, P_{t s 51}, P_{t s 52}, P_{t s 53}, P_{t s 54}, \\
& \left.P_{t s 6}, P_{t s 71}, P_{t s 72}, P_{t s 73}, P_{t s 74}, P_{t s 81}, P_{t s 82}\right\}
\end{aligned}
$$

In the formula,

$$
\begin{aligned}
& P_{t s 11}=\left\{I_{1} ; P_{11}\right\}, P_{t s 12}=\left\{P_{11} ; P_{11}, I_{15}, I_{16}\right\}, P_{t s 13}=\left\{P_{11} ; I_{15}\right\}, \\
& P_{t s 21}=\left\{I_{4}, I_{5}, I_{6}, P_{11}, P_{25} ; P_{22}\right\}, P_{t s 22}=\left\{I_{5}, P_{25} ; P_{23}\right\}, \\
& P_{t s 23}=\left\{P_{22}, P_{23} ; P_{21}\right\}, P_{t s 24}=\left\{P_{21} ; I_{11}\right\}, P_{t s 31}=\left\{P_{31} ; P_{32}\right\},
\end{aligned}
$$

$$
\begin{aligned}
& P_{t s 32}=\left\{I_{8}, I_{9}, P_{23}, P_{31} ; P_{33}\right\}, P_{t s 33}=\left\{P_{33} ; I_{13}\right\}, P_{t s 41}=\left\{P_{41} ; P_{43}\right\}, \\
& P_{t s 42}=\left\{P_{43}, P_{44} ; P_{45}\right\}, P_{t s 43}=\left\{P_{46} ; P_{48}\right\}, P_{t s 441}=\left\{P_{61} ; P_{63}\right\}, \\
& P_{t s 442}=\left\{P_{63} ; P_{62}\right\}, P_{t s 443}=\left\{P_{62} ; P_{63}\right\}, P_{t s 444}=\left\{P_{62} ; P_{64}\right\}, \\
& P_{t s 45}=\left\{P_{45}, P_{48}, P_{64} ; I_{17}\right\}, P_{t 551}=\left\{P_{51}, P_{57} ; P_{52}\right\}, \\
& P_{t s 52}=\left\{P_{51}, P_{52} ; P_{52}, P_{53}\right\}, P_{t s 53}=\left\{P_{52}, P_{53}, P_{57} ; P_{54}\right\}, \\
& P_{t s 54}=\left\{P_{54} ; I_{12}\right\}, P_{t s 6}=\left\{I_{2} ; I_{25}, I_{31}, P_{41}, P_{51}\right\}, \\
& P_{t s 71}=\left\{P_{11}, P_{71} ; P_{72}\right\}, P_{t s 72}=\left\{P_{72} ; P_{72}, P_{73}\right\}, \\
& P_{t s 73}=\left\{P_{71}, P_{73} ; P_{72}, P_{74}\right\}, P_{t s 74}=\left\{P_{72}, P_{74} ; I_{7}\right\}, \\
& P_{t s 81}=\left\{I_{6}, I_{10}, P_{21}, P_{53} ; P_{81}\right\}, P_{t s 82}=\left\{P_{81} ; I_{14}\right\} .
\end{aligned}
$$

\subsection{Integration of Petri Net Model}

Based on Petri net theory, in view of the MES system, a basic concept model between the actual problem and the system model is established. The model can be used to describe and analyze the MES system efficiently and quickly.

With the relevant theories of Petri net modeling, the Petri nets model of MES can be established as showed in Fig. (3). All the nodes elements in the graph are defined previously.

\section{PERFORMANCE ANALYSIS OF MES SYSTEM MODEL}

\subsection{Analysis of Model Complexity}

The MES system complexity is the complex characteristics in the elements, structure, status and process, etc. The relationship between the elements and structure reflects the static structure characteristics of the system, and the status and process characteristics depict the dynamic behavior of the system [7].

Setting the system complexity of the elements as $C_{n}$, the correlation complexity of the system as $C_{r}$, structural complexity as $C_{S}$. Since the influence of $C_{n}$ and $C_{r}$ on the structural complexity is different and the correlation coefficient between $C_{n}$ and the system structural complexity is $\lambda$, then [4]

$$
C_{S}=\lambda C_{n}+(1-\lambda) C_{r}
$$

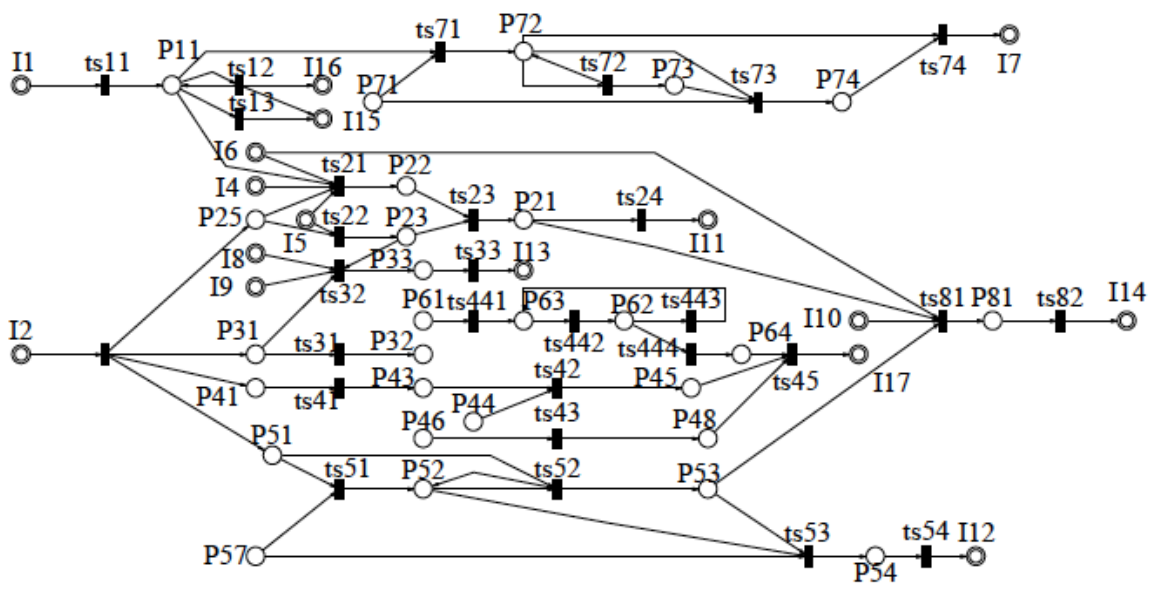

Fig. (3). Petri nets model $H_{1}$ of MES. 
In the MES Petri net model, the node is the element of the system, the influence of the node on system complexity can be described as the ratio between the number of in-arcs and out-arcs of the node and the total number of network connections arcs, that is

$\lambda=\frac{1}{|T|+|P|} \sum_{i=1}^{|T|+P \mid}\left(\frac{\left|\cdot x_{i}\right|+\left|x_{i}^{\bullet}\right|}{|X|}\right) \quad x_{i} \in \mathrm{X}$

$|X|$ represents the number of elements in set $X$.

When we use Petri nets model to describe the MES, the elements complexity $C_{n}$ is the function of location (Place) and transition nodes number in Petri model, set $C_{n}$ as

$$
\begin{aligned}
& C_{n}=\frac{1}{2}\left(\frac{|P|-1}{|P|}+\frac{|T|-1}{|T|}\right) \\
& \text { If }|P|=m,|T|=n, \text { then } \\
& C_{n}=\frac{1}{2}\left(\frac{m-1}{m}+\frac{n-1}{n}\right)=\frac{2 m n-m-n}{2 m n}
\end{aligned}
$$

In Petri net, when any position node $p_{i}$ is the enter location of two or more transition nodes $t_{j}(j=1,2, \cdots, n)$, as $p_{i} \in\left({ }^{\prime} t_{1} \cap{ }^{\cdot} t_{2} \cap \cdots\right),\left|p_{i}^{\bullet}>1\right|$, there is a conflict structure, denoted as $K_{i}=\left\langle p_{i}\left\{t_{1}, t_{2}, \cdots\right\}\right\rangle$. Conflict will cause the system behavior uncertainties, that is, the resources competition of the conflict structure makes it complicated to manage and control the system resources. So it is necessary to analyze the correlation complexity of the allocation, generation and use of the resource.

Definition $1^{[2]}$ Set the correlation complexity of any node $p_{i}$ in Petri net as $c_{p i}$, then

$$
c_{p i}=\frac{\left|p_{i}^{\bullet}\right|}{\left|\cdot p_{i}\right|+\left|p_{i}^{\bullet}\right|}
$$

From Definition 1, the correlation complexity $C_{P}$ of location set $P$ can be derived as:

$$
C_{P}=\frac{1}{|P|} \sum_{i=1}^{|P|} \frac{\left|p_{i}^{\bullet}\right|}{\left|\cdot p_{i}\right|+\left|p_{i}^{\bullet}\right|}=\frac{1}{m} \sum_{i=1}^{m} \frac{\left|p_{i}^{\bullet}\right|}{\left|\cdot p_{i}\right|+\left|p_{i}^{\bullet}\right|}
$$

Similarly, the correlation complexity $C_{T}$ of transition set in Petri net can be defined as

$$
C_{T}=\frac{1}{|T|} \sum_{j=1}^{|T|} \frac{\left|\cdot t_{j}\right|}{\left|t_{j}\right|+\left|t_{j}^{\bullet}\right|}=\frac{1}{n} \sum_{j=1}^{n} \frac{\left|\cdot t_{j}\right|}{\left|\cdot t_{j}\right|+\left|t_{j}^{\bullet}\right|}
$$

Then the entire MES Petri net structure complexity $C_{r}$ can be expressed as the average value of location set correlation complexity and transition set correlation complexity, as

$$
C_{r}=\frac{C_{P}+C_{T}}{2}
$$

From the Petri net model of MES system in Fig. (3) and the above definitions, the Petri net structural complexity of MES can be analyzed.

The number of elements in Place node set is $|P|=m=28$ $=29$

The set of directed arc in net is $|F|=88$

The number of the node set whose pre-set and post-set are not empty is $|X|=48$

According to the above parameters and related definitions, the value of $C_{n}, \lambda, C_{P}, C_{T}, C_{r}, C_{S}$ can be obtained respectively as Table $\mathbf{1}$.

Table 1. Result of experiments and theory.

\begin{tabular}{|c|c|c|c|c|c|c|}
\hline $\begin{array}{c}\text { Parameter } \\
\text { Name }\end{array}$ & $\boldsymbol{C}_{n}$ & $\lambda$ & $\boldsymbol{C}_{\boldsymbol{P}}$ & $\boldsymbol{C}_{\boldsymbol{T}}$ & $\boldsymbol{C}_{\boldsymbol{r}}$ & $\boldsymbol{C}_{\boldsymbol{S}}$ \\
\hline \hline Value & 0.96 & 0.03 & 0.62 & 0.48 & 0.55 & 0.56 \\
\hline
\end{tabular}

The structure correlation complexity of the Petri net is mainly determined by $C_{r}$, and the relationship between correlation structural condition and $C_{r}$ is shown in Table 2. By the value of $C_{r}$ in Table $\mathbf{1}$ and the criteria in Table 2, it is easy to see that MES system is a correlation system with certain complex structures.

Table 2. The relationship between Petri net correlation structural and $C_{r}$.

\begin{tabular}{|c|c|}
\hline The Range of $\boldsymbol{C}_{\boldsymbol{r}}$ & Correlation Complexity \\
\hline \hline 1 & Maximum \\
\hline 0 & Minimum \\
\hline $0<C_{r}<1 / 2$ & Correlation structure simple \\
\hline $1 / 2$ & Correlation structure ideal \\
\hline $1 / 2<C_{r}<1$ & Correlation structure complex \\
\hline
\end{tabular}

\subsection{Characteristics of the Model Node}

The main influence factor of an information system is the change of technology and demand environment. Therefore, scalable functionality is the basic requirements of modern enterprise information system. However, the function can't exist separately from the structure. So, we need to discuss the relationship among the elements of the various components of MES systems.

The influence of each node on the system can be analyzed and defined by the relationship between the node and other nodes in the system. And the coupling strength is used to measure the influence of each node at different locations on the system performance. This feature needs to be obtained by analyzing the relationship between one node and other nodes. Coupling strength is defined as follows: 
Definition $2^{[2]}$ Suppose $x_{i} \in P \cup T$ is any node on the net, $\left|\cdot x_{i}\right| \neq 0 \wedge\left|x_{i}^{\bullet}\right| \neq 0 . d\left(x_{i}\right)$ is the coupling strength of the node $x_{i}$ on the net, as

$$
d\left(x_{i}\right)=\frac{1}{2}\left(\frac{\left|M x_{i}\right|}{M_{S}}+\frac{\left|R_{\max }\left(x_{i}\right)\right|}{|X|}\right) .
$$

Among them, $M x_{i}$ is the set of all mesh correlated with the node $x_{i} ; R_{\max }\left(x_{i}\right)$ is the maximum one among the sum of $x_{i}{ }^{\prime} s$ pre-set and post-set and the set of other nodes related to node $x_{i}$ in the largest mesh; $|X|$ is the number of the node set whose pre-set and post-set are not empty.

Not considering the elements of the interface, according to the Petri net model on Fig. (3), the number of mesh $M_{S}$ in the figure is 13, illustrated in Fig. (4). Analyzing the information resource set and feature set without interface nodes in $H_{1}$, we get coupling strength of each node $d\left(x_{i}\right)$ and heavy weight node $x_{i}^{*}$ (Node's coupling strength is greater than the average of coupling strength) [6], as shown in Fig. (5).

Fig. (5) shows the average coupling strength for each node $D_{\Sigma}=0.08$, so we get the set of these key function nodes $T_{k}$ and the set of these key resource nodes $P_{k}$ :

$T_{k}=\left\{t_{s 21}, t_{s 22}, t_{s 23}, t_{s 32}, t_{s 51}, t_{s 52}, t_{s 53}, t_{s 6}, t_{s 72}, t_{s 73}, t_{s 81}\right\}$

$P_{k}=\left\{P_{21}, P_{22}, P_{23}, P_{25}, P_{31}, P_{51}, P_{52}, P_{53}, P_{71}, P_{72}, P_{73}\right\}$

Therefore, in the daily maintenance and adjustment process, for the above key functions nodes and key resource nodes we should try to avoid or reduce the appropriate modifications to ensure the stability and reliability of the system operation.

\subsection{The Core Layer of Petri Net and Reliability Analysis}

For any information system applied to manufacturing, there must be one or more elements playing a decisive role on the system structure and performance. Under normal cir- cumstances, we map the Petri net model of the MES systems as two types of undirected network diagram with weighted nodes and sides at the same time. According to the definition in [2] literature, we can analyze the MES Petri core network and the reliability of the system by solving the kernel and kernel degree of directed network system in the MES Petri network.

The transition node directed network graph $D_{t}$ and the place node directed network graph $D_{p}$ of MES information resource configuration model is shown in Fig. (6).

As shown in Fig. (6), the weighted kernel degree and weighted kernel of the two weighted undirected net graphs $G_{t}$ and $G_{p}$ corresponding with $D_{p}$ and $D_{t}$ are as follows:

$$
\begin{aligned}
& h\left(G_{t}\right)=1, \delta^{*}\left(G_{t}\right)=\left(\left\{t_{s 11}, t_{s 71}, t_{s 74}, t_{s 6}, t_{s 21}, t_{s 23}, t_{s 81}, t_{s 51}, t_{s 52}\right\},\right. \\
& \left.\left\{t_{s 11} t_{s 71}, t_{s 71} t_{s 74}, t_{s 6} t_{s 21}, t_{s 21} t_{s 23}, t_{s 23} t_{s 81}, t_{s 6} t_{s 51}, t_{s 51} t_{s 52}, t_{s 52} t_{s 81}\right\}\right) \\
& h\left(G_{p}\right)=1, \delta^{*}\left(G_{p}\right)=\left(\left\{P_{11}, P_{72}, P_{25}, P_{22}, P_{21}, P_{51}, P_{52}, P_{53}\right\},\left\{P_{11} P_{72},\right.\right. \\
& \left.\left.P_{11} P_{22}, P_{25} P_{22}, P_{22} P_{21}, P_{51} P_{52}, P_{52} P_{53}\right\}\right)
\end{aligned}
$$

The weighted kernel and the weighted kernel degree of MES are

$$
\begin{aligned}
& h(H)=h\left(G_{t}\right)+h\left(G_{p}\right)=1+1=2 \\
& \delta^{*}(H)=\delta^{*}\left(G_{t}\right) \cup \delta^{*}\left(G_{p}\right)=\left(\left\{t_{s 11}, t_{s 71}, t_{s 74}, t_{s 6}, t_{s 21}, t_{s 23}, t_{s 81},\right.\right. \\
& \left.t_{s 51}, t_{s 52}, t_{s 81}, P_{11}, P_{72}, P_{25}, P_{22}, P_{21}, P_{51}, P_{52}, P_{53}\right\},\left\{t_{s 11} t_{s 71}, t_{s 71} t_{s 74},\right. \\
& t_{s 6} t_{s 21}, t_{s 21} t_{s 23}, t_{s 23} t_{s 81}, t_{s 6} t_{s 51}, t_{s 6} t_{s 52}, t_{s 51} t_{s 52}, t_{s 52} t_{s 81}, P_{11} P_{72}, P_{11} P_{72}, \\
& \left.\left.P_{25} P_{22}, P_{22} P_{21}, P_{51} P_{52}, P_{52} P_{53}\right\}\right)
\end{aligned}
$$

The core of the Petri net model described by $\delta^{*}(H)$ is showed in Fig. (7).

Fig. (7) is the more central network $\mathrm{H}_{2}$ relative to Fig. (3). In the process of model design and test implementation, we should ensure the reachability and soundness of this level network firstly, then we can design a reliable system more efficient and fast.

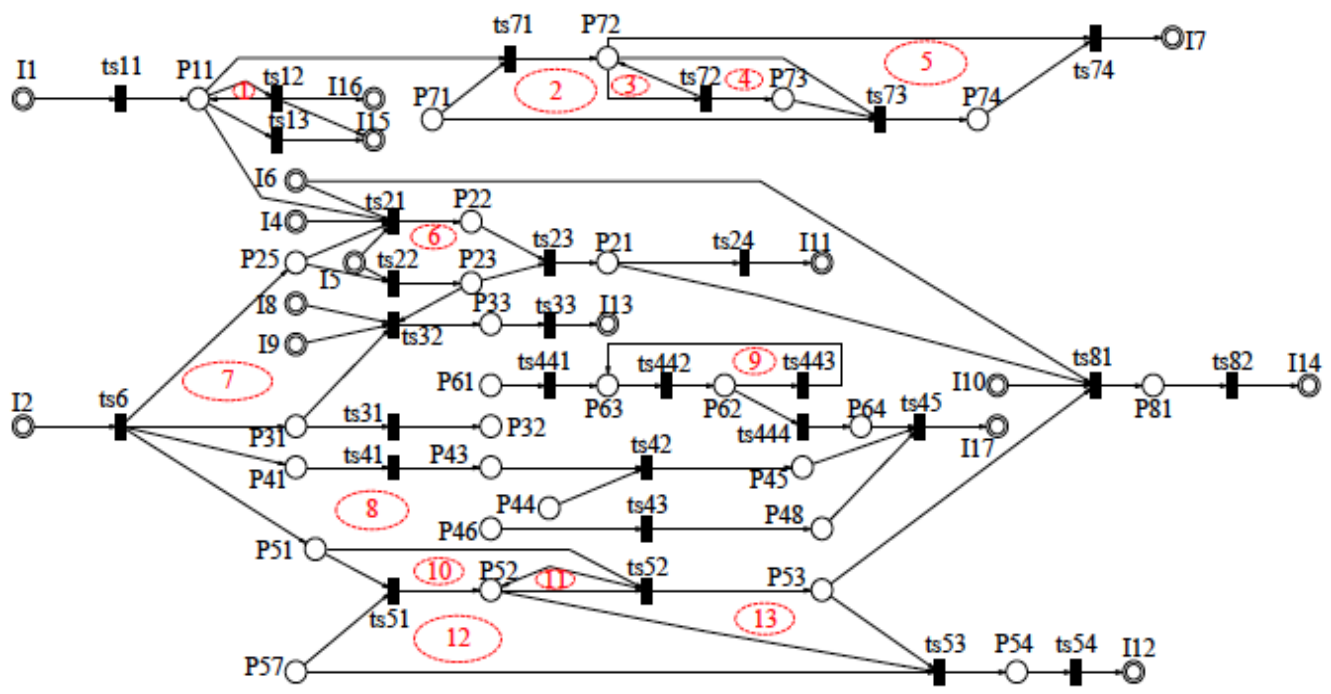

Fig. (4). Meshes in the Petri net model of MES. 

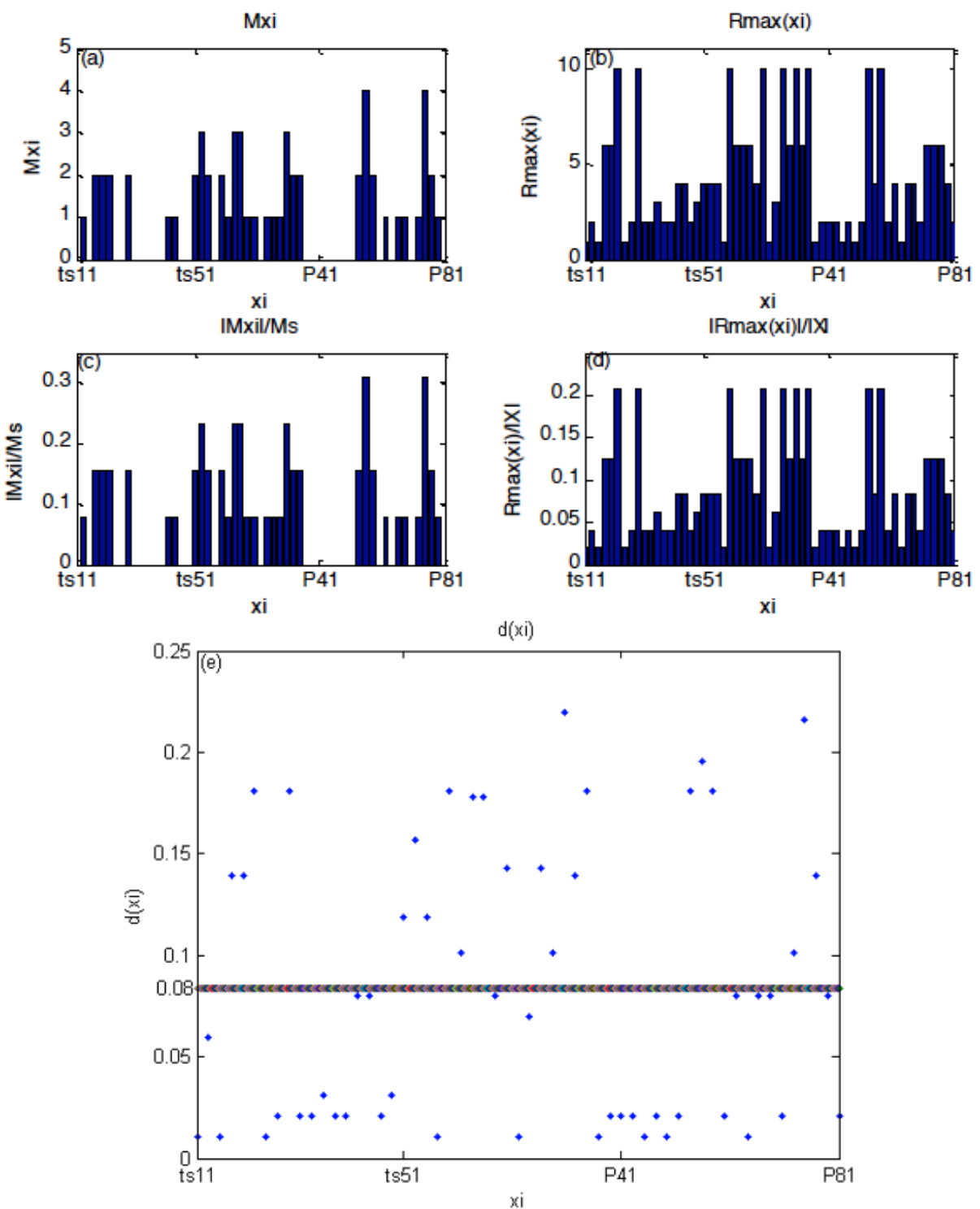

Fig. (5). (a) The mesh set of each node; (b) The sum of pre-set \& post-set; (c) The mesh set weight of each node; (d) The weight of the sum of pre-set \& post-set; (e ) Coupling strength of each node.
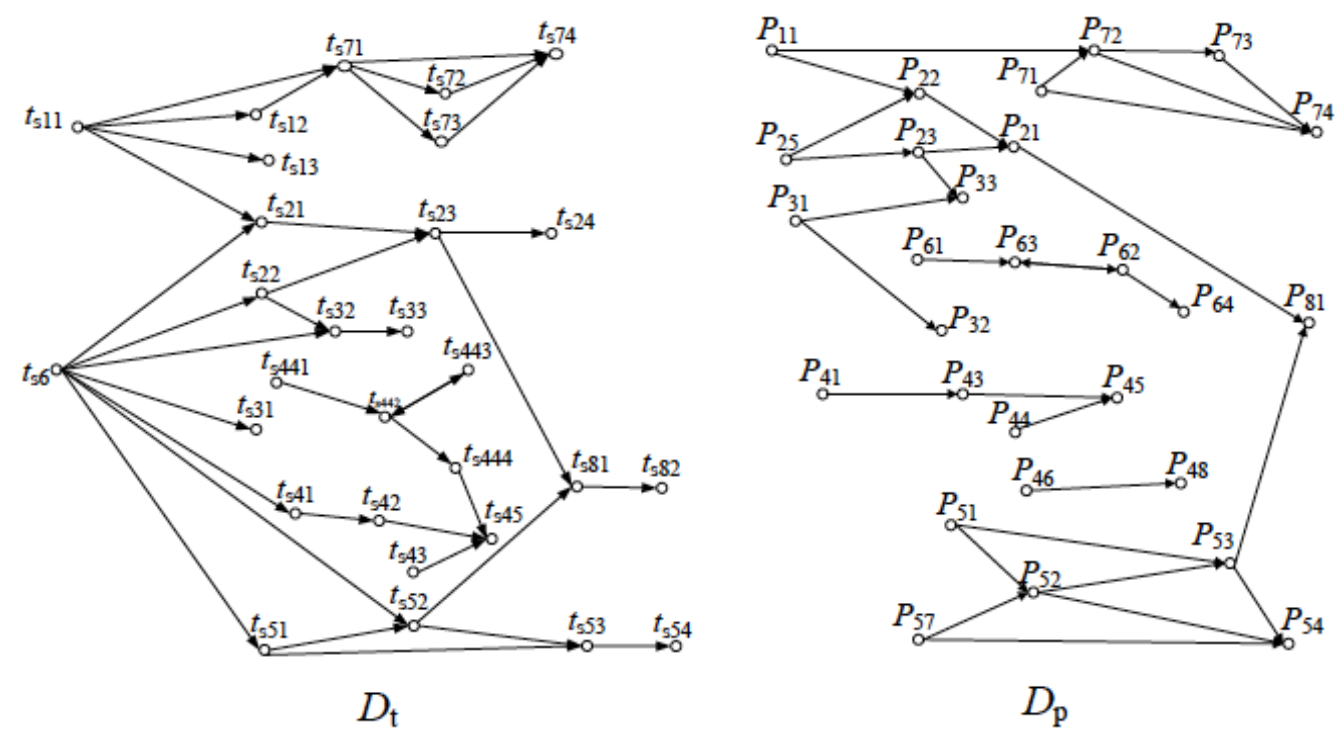

Fig. (6). Two types of directed network graphs of MES resource allocation model. 


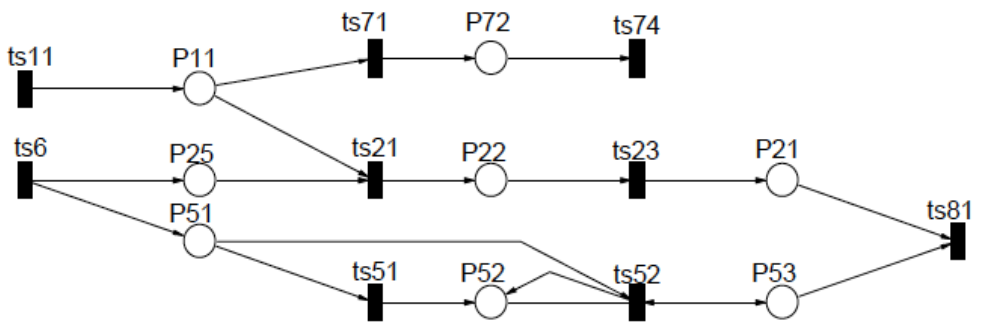

Fig. (7). The core layer network model H2 of MES.

If we do further analysis to network $H_{2}$, we can get the "core" of the core layer: $H_{3}=\delta^{*}\left(H_{2}\right)$, as shown in Fig. (8). Its practical significance is the analysis reports about the system output production when the system inputs basic data.

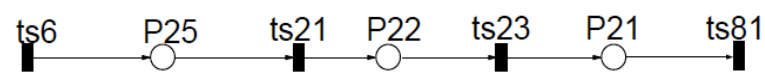

Fig. (8). The highest core network diagram $\mathrm{H}_{3}$ of MES.

The reliability of the system is one of the important indicators of system. For the system composed with various elements, the reliability of the system can be measured with the reliability of the various elements. Set the reliability of the system as $R_{s}(t)$, the reliability of the various elements as $R_{i}(t),(i=1,2, \cdots, n)$, then the relationship between the series system and the parallel system can be shown as follows:

$R_{s}(t)=\prod_{i=1}^{n} R_{i}(t)$

$R_{s}(t)=1-\prod_{i=1}^{n}\left(1-R_{i}(t)\right)$

For general redundant system, the two same devices parallel to form a unit, then the equipment reliability with dual redundant can be obtained by (12):

$$
R_{r}(t)=1-(1-R(t))(1-R(t))=2 R(t)-R^{2}(t)
$$

Assume that the important degree of each network layer node is $I_{j}$, the important degree of the most basic network node $I_{1}$ is 1 , and the node important degree add 1 when the network increases one layer, so $I_{j}=j$, and the important degree of all nodes $I_{\mathrm{S}}$ is

$I_{S}=\sum_{j=1}^{m} I_{j}\left|H_{j}-H_{j+1}\right|$ is

The reliability allocation ratio $K_{j}$ for each network layer

$$
K_{j}=\frac{I_{j}\left|H_{j}-H_{j+1}\right|}{I_{S}} \times 100 \%
$$

According to the definition of core degrees, we can see that each layer of network structure maintains its own connectivity. The operation of one layer cannot be affected by the fault of any another layers, so we assume that the reliability of each network layer is $R_{i}(t)$, and then the overall reliability of the system is expressed approximately as

$$
R_{s}(t)=\sum_{j=1}^{m} R_{i}(t) K_{j}
$$

The reliability $R_{i}(t)$ of each network layer can be calculated depending on the situation by (10) and (11).

Assume that the reliability of 57 nodes running separately in Fig. (4) are $R_{i}(t)=98 \%(i=1,2, \ldots, 57)$, then the Petri net model of MES can be decomposed into three network layer: $H_{1}$ in Fig. (3), $H_{2}$ in Fig. (7) and $H_{3}$ in Fig. (8), the corresponding important degree $I_{j}$ and reliability allocation proportion $K_{j}$ as shown in Table 3 .

According to calculation results in Table $\mathbf{3}$, we obtained the system required equipment and corresponding reliability comparison table based on four different redundancy schemes, as showed in Table 4.

Table 3. Important degree and reliability comparison of three network layers.

\begin{tabular}{|c|c|c|c|}
\hline Level & $\boldsymbol{H}_{\mathbf{1}}$ & $\boldsymbol{H}_{\mathbf{2}}$ & $\boldsymbol{H}_{\mathbf{3}}$ \\
\hline \hline$H_{\mathrm{j}}|-| H_{\mathrm{j}+1} \mid$ & 40 & 10 & 7 \\
\hline$I_{j}$ & 1 & 2 & 3 \\
\hline$K_{j}$ & $49.3 \%$ & $24.7 \%$ & $26.0 \%$ \\
\hline
\end{tabular}

From Table 4, we know that the system reliability order of the four system redundancy schemes are (2)(3)(4)(1) from high to low. Considering the case of less equipment and high reliability, our choice is Scheme (4). In case of allowed charges, we choose Scheme (3).

As can be seen from reliability analysis process of the system, the conclusive influence on the reliability of the system is not only the reliability of each node, but also the importance and reliability of each core level in the system. So, before the system design, we should design a preliminary system model according to the actual demand of function and performance, then we should analyze and improve the system core layer further to meet the requirements of reliability and funds.

\section{CONCLUSION}

Based on the functional structure of MES, a Petri net model is established according to the relationship between the function nodes and the resource nodes, then the reliability and complexity of the model was analyzed according to reliability analysis theory and graph theory methods. 
Table 4. Important degree and reliability comparison of three network layers.

\begin{tabular}{|c|c|c|c|c|c|}
\hline Redundancy Scheme & Device Number $n$ & $\begin{array}{c}\text { Reliability Degree } \\
R_{\mathrm{S}}(\%)\end{array}$ & Scheme Comparison & $\begin{array}{c}\text { Added Value of } \\
\text { Equipment Number } \\
(\%)\end{array}$ & $\begin{array}{c}\text { Added Value of } \\
\text { Reliability (\%) }\end{array}$ \\
\hline (1) No node is redundant & 57 & 0.03 & & & \\
\hline (2) All nodes are redundan & 114 & 97.5 & (2): (1) & 100 & 50.4 \\
\hline \multirow{2}{*}{$\begin{array}{c}\text { (3) Nodes in } \mathrm{H}_{2} \text { are redun- } \\
\text { dant }\end{array}$} & \multirow{2}{*}{74} & \multirow{2}{*}{72.2} & (3):(1) & 29.8 & 11.4 \\
\hline & & & (3):(2) & -35.0 & -25.9 \\
\hline \multirow{3}{*}{$\begin{array}{c}\text { (4) Nodes in } \mathrm{H}_{3} \text { are redun- } \\
\text { dant }\end{array}$} & \multirow{3}{*}{64} & \multirow{3}{*}{68.0} & (4): 1 & 12.3 & 4.9 \\
\hline & & & (4):(2) & -43.8 & -30.2 \\
\hline & & & (4):(3) & -13.5 & -5.8 \\
\hline
\end{tabular}

From the analysis results, we can see that the MES system model has certain complexity, and the MES Petri net model can make a good description of the relationship between each functional component of MES, and it provides a good model design basis for the early development of MES software. For the reliability analysis, the main characteristics of the model can be clearly presented by the network core layer, but the core layer $\mathrm{H}_{2}$ still has some limitation, and it cannot reflect the characteristics of the core layer from the best angle. So, in the future study, we need to improve $\mathrm{H}_{2}$, deeply to maximize the response model characteristics and increase the model reliability further.

\section{CONFLICT OF INTEREST}

The authors confirm that this article content has no conflict of interest.

\section{ACKNOWLEDGEMENTS}

Declared none.

\section{REFERENCES}

[1] X. G. Li, C. B. Li, F. Liu, and L. L. Li, "Modeling and quantification methods for carbon emission in machine tools manufacturing processes based on Petri nets," Computer Integrated Manufacturing Systems, vol. 18, no. 12, pp. 2723-2735, 2012.

[2] J. Wang, "Analysis of hierarchy structure in information system based on the Petri Nets," Computer Engineering and Applications, no. 4, pp. 89-91, 2002

[3] G. Zhang, J. Liu, H. Ge, and H. Zhang, "Modeling for assemb-ly reliability based on generalized stochastic Petri Nets," Computer Integrated Manufacturing Systems, vol. 18, no. 3, pp. 507-512. 2012.

[4] J. Wang, and R. Gan, "Information system modeling based on Petri Nets," Chinese Journal of Management Science, vol. 8, no. 2, pp. 20-27, 2000.

[5] Q. Wang, Service-Oriented Manufacturing Execution System Theory and Application, Zhejiang University Press, Hangzhou, 2012.

[6] M. J. Rabbani, F. M. Ahmad, J. Baladi, Y. A. Khan, and R. A. Naqvi, "Modeling and simulation approach for an industrial manufacturing execution system," IEEE 3rd International Conference on System Engineering and Technology (ICSET 2013), no. 8, pp. 2631, 2013.

[7] O. Djamila, and P. Sanja, "A survey of dynamic scheduling in manufacturing systems," Intelligent Systems Design and Applications, vol. 12, no. 8, pp. 417-431, 2009.

[8] C. Cao, N. Wang, and Y. Wang, "On UML and Petri Net based modeling of integratable manufacturing executing system," $\mathrm{Me}$ chanical Science and Technology, vol. 23, no. 9, pp. 1093-1102, 2004.

(C) Taiping and Peisi; Licensee Bentham Open.

This is an open access article licensed under the terms of the Creative Commons Attribution Non-Commercial License (http://creativecommons.org/licenses/by-nc/3.0/) which permits unrestricted, non-commercial use, distribution and reproduction in any medium, provided the work is properly cited. 